Correspondence

Michael Ellis

michael.ellis@uaeu.ac.ae

Received 3 May 2009

Accepted 9 July 2009

\section{A safety and feasibility study comparing an intermittent high dose with a daily standard dose of liposomal amphotericin B for persistent neutropenic fever}

\author{
Michael Ellis, ${ }^{1,2}$ Roos Bernsen, ${ }^{2}$ Hussein Ali-Zadeh, ${ }^{1,3}$ \\ Jörgen Kristensen, ${ }^{1,3}$ Ulla Hedström, ${ }^{1,4}$ Lazaros Poughias, ${ }^{5}$ Mark Bresnik, ${ }^{6}$ \\ Awad Al-Essa ${ }^{1}$ and David A. Stevens ${ }^{7}$ \\ ${ }^{1}$ Department of Medicine, Faculty of Medicine and Health Sciences, United Arab Emirates \\ University, Al Ain, UAE \\ ${ }^{2}$ Department of Community Medicine, Faculty of Medicine and Health Sciences, United Arab \\ Emirates University, Al Ain, UAE \\ ${ }^{3}$ Department of Oncology, Tawam-Johns Hopkins Hospital, Al Ain, UAE \\ ${ }^{4}$ Department of Medicine, Al-Ain Hospital, Al Ain, UAE \\ ${ }^{5}$ International Medical Affairs, Gilead Sciences, London, UK \\ ${ }^{6}$ Gilead Sciences, Foster City, CA, USA \\ ${ }^{7}$ Santa Clara Valley Medical Center, San Jose, CA, USA
}

A high intermittent dose regimen (group A: $10 \mathrm{mg} \mathrm{kg}^{-1}$ on day $1,5 \mathrm{mg} \mathrm{kg}^{-1}$ on days 3 and 6) was compared with standard dosing (group B: $3 \mathrm{mg} \mathrm{kg}^{-1}$ per day for 14 days) of liposomal amphotericin $B(L A B)$ for empirical treatment of persistent febrile neutropenia. A total cumulative dose of $1275 \mathrm{mg}$ (group A) and $2800 \mathrm{mg}$ (group B) was administered. Infusion-related adverse drug events, mainly rigors/chills, occurred more frequently with group $A(11 / 45,24 \%$ infusions) than with group $B(12 / 201,6 \%$ infusions) $(P=0.002)$, which extended the mean infusion time by $20 \mathrm{~min}(P=0.001)$. Creatinine levels were similar in the two regimens: the $\mathrm{A}: \mathrm{B}$ ratio of the area under the curve for creatinine (AUC CREATININE$_{\text {f }}$ for days $2-7$ was $1.09(P=0.27)$ and for days 2-14 was $1.05(P=0.51)$. Rises in creatinine were mild (clinical toxicity criteria 1 ) in all patients with elevations. Hypokalaemia tended to be less severe in group $A$ with a lower proportion of

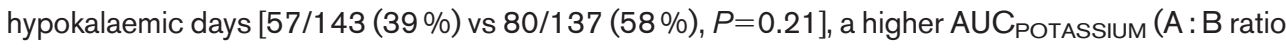
of 1.06, $P=0.12$ ), a lower proportion of patients with hypokalaemia at the end of study (10 vs $61 \%$, $P=0.01)$ and fewer potassium-supplemented days [12/210 (6\%) vs 41/210 (19.5\%), $P<0.1]$. There were mildly elevated median levels of serum bilirubin, alanine aminotransferase, aspartate aminotransferase and alkaline phosphatase, which were similar for the two regimens and were usually associated with other co-existing co-morbid conditions. The AUC for these enzymes was also similar in the two groups. No patient had discontinuation of the study drug due to toxicity. Composite success was identical for each regimen (11/15 patients, $73 \%$ ). Three of the fifteen patients in group $\mathrm{B}$ and none in group $\mathrm{A}$ developed invasive fungal infections (IFls). $\beta$-D-Glucan levels were similar in both groups for patients without an IFI [AUC GLUCAN $_{\text {of }} 362$ and $683(P=0.36)$ for groups $\mathrm{A}$ and $\mathrm{B}$, respectively]. The rate of defervescence was similar for each regimen $(P=0.75)$. This feasibility study suggests that a short intermittent high-dose course of $10 / 5 / 5 \mathrm{mg} \mathrm{LAB} \mathrm{kg}^{-1}$ on days 1, 3 and 6 may be as safe and effective as a standard 14 day course of $3 \mathrm{mg} \mathrm{kg}^{-1}$ per day, with drug-acquisition cost savings and reduced drug exposure. A larger study is indicated for confirmation of this. the curve; BDG, $\beta$-D-glucan; CTC, clinical toxicity criteria; EORTC, European Organisation for Research and Treatment of Cancer; HCV, hepatitis C virus; HSV, herpes simplex virus; IATCG, International Antimicrobial Therapy Cooperative Group; IFI, invasive fungal infection; IPA, invasive pulmonary aspergillosis; IRADE, infusion-related adverse drug event; LAB, liposomal amphotericin B; MAP, mean arterial pressure; MRC WPLA, Medical Research Council Working Party on Leukaemia in Adults; NCI, National Cancer Institute; PFN, persistent febrile neutropenia; ULN, upper limits of normal.

Tables and figures of additional data are available as supplementary material with the online version of this paper. 


\section{INTRODUCTION}

Early, empirical antifungal treatment of patients with haematological malignancy and persistent febrile neutropenia (PFN) reduces the risk of invasive fungal infection (IFI) (EORTC IATCG, 1989; Garey et al., 2006; Greene et al., 2007; Goldberg et al., 2008; Patterson et al., 2000).

Liposomal amphotericin B (LAB) (AmBisome) is used for empirical therapy because of its wide antifungal spectrum, reduced toxicity and clinical efficacy (Goldberg et al., 2008; Lanternier \& Lortholary, 2008; Moen et al., 2009; OstroskyZeichner et al., 2003; Takemoto et al., 2006a; Walsh et al., 1999). The recommended regimen is $3 \mathrm{mg} \mathrm{kg}^{-1}$ per day on consecutive days given until defervescence or for a minimum of 14 days (Hughes et al., 2002). This approach is expensive and time-consuming, and risks adverse drug events (ADEs) (Marr, 2008; Masiá Canuto \& Rodero, 2002; Saliba \& Dupont, 2008).

The efficacy of amphotericin B is partly related to its high peak serum concentration/MIC ratio (Takemoto et al., 2006b), which is more readily achievable with the liposomal formulation (Bekersky et al., 2002). Adequate and sustained LAB deposition in fungal target tissues is also a factor related to antifungal activity (Adler-Moore \& Proffitt, 2003; Collette et al., 1991; Smith et al., 2007). When LAB is administered at higher doses, more amphotericin B is deposited in tissues, exerting increased anti-fungal activity (Adler-Moore \& Proffitt, 2003; Demartini et al., 2005; Garcia et al., 2000; Townsend et al., 2001; Vogelsinger et al., 2006). Tissue levels have been shown to remain above the MIC for many fungi for at least 1-6 weeks (Smith et al., 2007).

These observations provide a rationale for investigating an initial early administration of a divided high-dose LAB over the first 5 days of PFN. It is not known whether this approach would improve efficacy because of higher initial serum levels and targeted tissue loading. Indeed, a recent study failed to demonstrate improved efficacy of a higher dose of LAB in treating established IFIs (Cornely et al., 2007). It is also uncertain whether efficacy will be diminished due to intermittent dosing and lower cumulative dose (AdlerMoore \& Proffitt, 2008; Walsh et al., 2001; Francis et al., 1994; Olson et al., 2006). Furthermore, it is not known whether safety (e.g. renal function) will be compromised due to higher drug exposures with each dose, which in turn could lead to treatment discontinuation, or whether safety will be better due to longer dosing intervals, a shorter treatment course and a lower cumulative dose (Bates et al., 2001). This study was conducted to establish the safety and feasibility of using a high, intermittent, short course of LAB.

\section{METHODS}

\section{Patients}

The primary objective of this study was to assess the safety of administering a high intermittent dose of LAB to patients with PFN, by measuring common toxicities. The secondary objective was to assess the feasibility of using LAB in such a way, in terms of efficacy of success using widely used measurements of composite success, the emergence of IFIs and defervescence.

The study design was an open, randomized clinical trial. It was approved by the Al Ain Medical District Human Research Ethics Committee. Recruited subjects provided informed, written consent.

Patients admitted to the haematology ward of Tawam-Johns Hopkins Hospital with haematological malignancy received appropriate induction or consolidation chemotherapy according to international guidelines (See Supplementary Table S1, available with the online journal) (Coiffier et al., 2002; Farag et al., 2005; MRC WPLA, 2006). Those developing (i) fever (a single oral temperature of $\geqslant 38.3{ }^{\circ} \mathrm{C}$, or a temperature of $\geqslant 38.0{ }^{\circ} \mathrm{C}$ for $\geqslant 1 \mathrm{~h}$; Hughes et al., 2002) and (ii) neutropenia $\left(<0.5 \times 10^{9}\right.$ neutrophils $\left.1^{-1}\right)$ for 3 days together with (iii) negative bacteriological blood cultures, (iv) an absence of response (defervescence) to broad-spectrum antibiotics (piperacillintazobactam) for 3 days, and (v) no clinical features of target organ infection were classified as PFN and randomized into group A or group B for the study, by means of a Pharmacy Department (TawamJohns Hopkins Hospital, Al Ain, UAE) computer-generated code.

In addition to the five preceding criteria, eligibility required: (i) informed written consent; (ii) absence of known established IFIs at baseline; (iii) no polyene drug administration for the previous 60 days; (iv) baseline liver enzymes and creatinine less than tenfold and twofold the upper limits of normal (ULN), respectively; and (v) no previous serious adverse reaction to amphotericin $B$.

Group A patients received $10 \mathrm{mg} \mathrm{LAB} \mathrm{kg}{ }^{-1}$ on day 1 , followed by $5 \mathrm{mg} \mathrm{LAB} \mathrm{kg}{ }^{-1}$ on days 3 and 6. Group B patients received $3 \mathrm{mg} \mathrm{LAB}$ $\mathrm{kg}^{-1}$ day $^{-1}$ for 14 days. LAB was reconstituted following the manufacturer's instructions in $5 \%$ glucose and infused over $2 \mathrm{~h}$. Paracetamol with diphenhydramine was administered for clinical toxicity criteria (CTC) 1 infusion-related chills/rigors, and hydrocortisone with pethidine for CTC2-4 reactions [National Cancer Institute (NCI) Cancer Therapy Evaluation Programme Common Toxicity Criteria, version 2.0, 1998 (NCI, 1998)].

Both groups of patients were studied for 14 days. Vital signs were recorded every $6 \mathrm{~h}$ as follows: temperature was measured sublingually with a routine device (IVAC TEMP.PLUS II, model 2080); blood pressure was measured with the subject in the recumbent position using a Phillips non-invasive cuff and monitor (M1573A); and pulse rate was measured at the radial pulse location over a period of $1 \mathrm{~min}$. Maximum and minimum mean arterial pressures (MAPs) were calculated using the formula $\mathrm{MAP}=[(2 \times$ diastolic blood pressure $)+$ systolic blood pressure]/3.

Subjects were assessed on a daily basis for LAB-associated ADEs, including chills/rigors, wheezing, hypotension, chest pain, dyspnoea during the infusion period or within $6 \mathrm{~h}$ afterwards, and rash or other unexpected incident during the 14 day observation period. The investigator decided whether the event was causally related to the infusion. It was not possible to assess haemoglobin concentrations, white cell counts or platelets for LAB-related toxicity because of the substantially greater and complex confounding influence that chemotherapy has on these variables.

Biochemical and haematological parameters were measured daily and related to the local laboratory normal reference standard values (Supplementary Table S2 available with the online journal). Daily physical examination was performed. Common toxicity criteria of the NCI were used to grade the clinical and biochemical abnormalities recorded (NCI, 1998) (Supplementary Table S2).

Computed tomography scans of the abdomen and chest were performed on day 1 and weekly during the study period. Blood was 
sampled on alternate days for $\beta$-D-glucan (BDG) concentration with a Fungitell assay as described previously (Ellis et al., 2008). A value of $\geqslant 80 \mathrm{pg}$ BDG $\mathrm{ml}^{-1}$ was taken as a positive BDG test. An IFI was defined using the European Organisation for Research and Treatment of Cancer/Mycoses Study Group revised criteria (De Pauw et al., 2008). The occurrence of a serious adverse event or development of an IFI after study enrolment necessitated withdrawal from the study.

A five-point composite score, as described previously, was used to determine the response. A positive response comprised all of the following: survival to $\geqslant$ day 7 after completion of study drug administration, resolution of fever in neutropenia, successfully treated baseline IFI if present, absence of breakthrough IFI and absence of study drug termination due to toxicity/inefficacy (Walsh et al., 2004).

\section{Statistics}

Sample size. We assumed that regimen A might be more nephrotoxic, defined as a rise in serum creatinine from a baseline of at least 1.5 -fold, and predicted that $5 \%$ of patients in group B and $50 \%$ in group A would develop a creatinine rise $\geqslant 1.5$ ULN on at least 1 day during the 14 day study period (Cornely et al., 2007; Deray, 2002; Saliba \& Dupont, 2008; Walsh et al., 2001). Based on these single proportions, we calculated that 15 patients were needed in each group to demonstrate, using a one-sided test, at least this difference with a power of $80 \%$ and a significance level of 0.05 (Fleiss et al., 2003).

Statistical analysis. Comparison of data between the two groups was performed for the entire study period of 14 days, as well as the initial 7 days, in order to capture and compare data during periods of actual exposure to LAB (days $2-13$ in group B, days $2-7$ in group A). For blood parameters and vital signs, we estimated cubic curves over the 14 day period in order to compensate for missing values and also to achieve some data smoothing. Then we calculated for each blood parameter and vital sign the area under the curve (AUC) for days 2-7 and days $2-14$ by adding the predicted values obtained by the cubic curve fitting. The following analysis was performed for each parameter and each vital sign: a linear regression with the appropriate AUC as dependent variable and treatment group, and the appropriate baseline value as independent variables. To compensate for outliers, logarithmic transformation of the data preceded the regression analysis. In all analyses, only subjects with at least four measurements taken in the 2 week treatment period were used.

Additional tests were performed to compare the duration of infusion time between the two study groups: a mixed linear regression to test the difference in duration and a mixed logistic regression to test the difference in percentage of extended infusions (both models took the multilevel structure of the data into account). Mixed logistic regression was also used to compare the percentage of blood samples revealing hypokalaemia. The difference in number of adverse events during infusions was tested with Poisson regression and the difference in number of days with potassium supplementation with the MannWhitney test. Finally, the difference in percentage of patients with moderate or severe adverse events during infusions, the difference in percentage of patients with hypokalaemia on days 7 and 14, and the difference in percentage of patients with a positive BDG test in the absence of an IFI at the end of the study were tested with Pearson's $\chi^{2}$ test with continuity correction, or with Fisher's exact test if the sizes were too small for $\chi^{2}$ analysis.

\section{RESULTS}

Thirty patients were randomized. Their characteristics are summarized in Table 1. Age, sex, co-morbidities, haematological disease, duration of prior neutropenia and fever, and systemic toxicity features (MAPs and temperature) were similar in the two groups. One patient in group A, despite being asymptomatic at enrolment, was found to have invasive pulmonary aspergillosis (IPA) at day 1 following a computed tomography scan. Growth factors were received by $13 / 15$ and $12 / 15$ patients in groups $\mathrm{A}$ and

Table 1. Patient and baseline characteristics

Note that one patient in each group had fewer than 3 days with $<0.5$ neutrophils $1^{-1}$, but had $>0.5$ to $<1.0$ neutrophils $1^{-1}$ prior to inclusion.

\begin{tabular}{|lcc|}
\hline Characteristic & Group A $(\boldsymbol{n}=\mathbf{1 5})$ & Group B ( $\mathbf{n = 1 5})$ \\
\hline Age in years: median (range) & $36(17-55)$ & $38(18-55)$ \\
Male : female (no. of patients) & $13: 2$ & $9: 6$ \\
Asian : other race (no. of patients) & $12: 3$ & $13: 2$ \\
AML (no. of patients) & 9 & 11 \\
ALL (no. of patients) & 4 & 3 \\
Lymphoma (no. of patients) & 2 & 1 \\
Co-morbid medical conditions (no. of patients) & $4{ }^{*}$ & 0 \\
Antifungals within 30 days (no. of patients) & 0 & $8(1-16)$ \\
Neutropenic days to enrolment: median (range) & $8(1-15)$ & $4(1-6)$ \\
Febrile neutropenic days to enrolment: median (range) & $4(2-8)$ & $39.2(37.2-40.1)$ \\
Maximum temperature ( ${ }^{\circ}$ C): median (range) & $38.8(37.4-39.8)$ & $94(77-107)$ \\
Maximum MAP (mm Hg): median (range) & $90(75-106)$ & $75(63-92)$ \\
Minimum MAP (mm Hg): median (range) & $74(57-92)$ & 12 \\
Other nephrotoxic drugs (no. of patients) & 12 & \\
\hline
\end{tabular}

ALL, Acute lymphoblastic leukaemia; AML, acute myelocytic leukaemia.

${ }^{\star}$ Mental disability (1), diabetes mellitus and HCV infection (1), diabetes mellitus, hypertension and ischaemic heart disease (1), diabetes mellitus and hypertension (1).

$\dagger$ Diabetes mellitus (1), hepatitis B virus infection (1), hypertension and hypothyroidism (1), asthma and systemic lupus erythematosus (1). 
$\mathrm{B}$, respectively, and corticosteroids were given to $4 / 15$ and $3 / 15$ patients, respectively. The neutropenic profile was similar for both treatment groups over the 14 days of the study period (Supplementary Fig. S1 available with the online journal).

The median cumulative dose $\mathrm{kg}^{-1}$ and total dose of LAB administered per patient for group A were $20.4 \mathrm{mg} \mathrm{kg}^{-1}$ (range 15.6-21.4) and $1275 \mathrm{mg}$ (range 800-2000), respectively, and for group B were $42.6 \mathrm{mg} \mathrm{kg}^{-1}$ (range 39.0-56.1) and $2800 \mathrm{mg}$ (range 1750-4200), respectively. There was a total of 45 daily infusions in group A and 201 in group B. All intended infusions were given in group A but 9/210 were missed in group $\mathrm{B}$ because of changes in treatment due to the development of an IFI (1 patient: 7 infusions) or patient refusal (2 patients: 1 infusion each). Infusion times were prolonged in group A from the intended duration of 120 min. Mean \pm SD infusion times were $142.3 \pm 43.5$ (group A) compared with $123.9 \pm 15.9 \mathrm{~min}$ in group $\mathrm{B}(P=0.001)$. A total of 10 out of 45 infusions in group A were extended beyond $150 \mathrm{~min}(155-335 \mathrm{~min})$ for chills/rigors in 8 of the 10 patients and for technical problems with intravenous access in the remaining 2 patients, compared with $3 / 201$ infusions $(210-250 \mathrm{~min})$ in group B $(P<0.0005)$ for chills/rigors.

\section{Toxicity}

Infusion-related toxicity. A total of 23 distinct infusionrelated ADEs (IRADEs) among the 246 infusions (9\%) were documented, of which 21 were considered definitely to be drug-acquired. Full details are given in Supplementary Table S3 (available with the online journal). At least one IRADE was documented in seven patients ( $47 \%)$ in group A and six patients $(40 \%)$ in group B who experienced 11 and 12 separate events, respectively. The proportion of IRADEs among the total infusions administered overall to the 30 patients was 23/246 infusions (9\%) but occurred significantly more frequently in group A $[11 / 45$ infusions $(24 \%)]$ than in group B $[12 / 201$ infusions $(6 \%)](P=0.002)$. A total of 20 of the 23 IRADEs ( $86 \%$ ) occurred during the first 3 days of infusion: 17 on day 1,1 on day 2,2 on day 3 and 3 on day 4 or after.

Rigors/chills were the most commonly observed ADE: 4/15 patients in group A $(26 \%)$ and $3 / 15(20 \%)$ in group B. Three of the five reactions $(60 \%)$ in group A were moderately severe (CTC2), whilst all reactions in group B were mild (CTC1) $(P=0.46$, Fisher's exact test). All ADEs were managed successfully with diphenhydramine, corticosteroid or paracetamol and/or temporary (30 min) cessation of the infusion or reduction of the infusion rate, followed by its resumption. No patient had discontinuation of the drug for toxicity.

\section{Renal toxicity}

Serum creatinine. The median serum creatinine baseline (day 1) concentrations were similar in each group: $56 \mu \mathrm{mol}$ $1^{-1}$ (range 44-78) for group A and $59 \mu \mathrm{mol} \mathrm{l}^{-1}$ (range 4673) for group B (Fig. 1a; see Supplementary Table S4 available with the online journal for full details). In group A, these levels became slightly elevated over the infusion period and then fell progressively following the last study drug infusion, reaching values at day 9 similar to those at baseline. In contrast, creatinine concentrations in group B remained at or close to the baseline level throughout the entire study period, but showed a slight rise after day 10 .

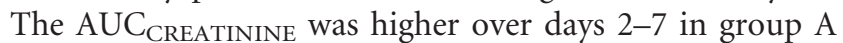
compared with group $\mathrm{B}$ patients $\left(\mathrm{AUC}_{\text {CREATININE }} \mathrm{A}: \mathrm{B}\right.$ ratio of 1.09) but this difference was not significant $(P=0.27)$. Over days $2-14$, the $\mathrm{AUC}_{\mathrm{CREATININE}}$ ratio was $1.05(P=0.51)$.

The number of patients whose serum creatinine concentrations became elevated above the ULN for at least 1 day over the entire 14 days was one in group A and two in group B (Fig. 1a, coloured lines). The maximum rise in creatinine in these patients was 1.2 times, 1.1 times and 1.2 times the ULN (CTC1), respectively. The patient in group A with an abrupt rise in creatinine at days 2-5 (Fig. 1a, coloured line) was receiving two other nephrotoxic drugs (vancomycin and gentamicin), and had severe sepsis and hypotension (blood pressure $88 / 40 \mathrm{mmHg}$ ) from extensive pan-mucositis. One of the two patients in group B with elevated creatinine had a severe drug (vancomycin) reaction, whilst the other had a pulmonary embolism.

At day 7, 15/15 patients (100\%) in group A and 12/13 $(92 \%)$ patients in group B had creatinine concentrations within the normal range. At day 14, these proportions were $10 / 10(100 \%)$ and $11 / 12(92 \%)$, respectively.

The number of patients receiving at least one nephrotoxic drug apart from LAB was 12/15 in each group, with the mean number received being 1.13 and 1.0 for groups $A$ and $B$, respectively. These were vancomycin ( 7 in group $A, 11$ in group B), teicoplanin (6 in group A, 2 in group B), aminoglycosides (2 in group A, 2 in group B). The scheduling of their administration over the study period was similar in both groups.

Serum potassium. The median potassium concentration at baseline was similar in both treatment groups: $3.4 \mathrm{mmol}$ $\mathrm{l}^{-1}$ (range 2.7-4.1) for group A and $3.9 \mathrm{mmol} \mathrm{l}^{-1}$ (range 3.3-4.4) for group B (Fig. 1b; see Supplementary Table S4 available with the online journal for full details). However, at baseline, a higher proportion of patients with hypokalaemia was documented in group A compared with group B (54 vs $31 \%)(P=0.14)$.

A total of 14 out of 15 group A patients (93\%) and 11/13 $(85 \%)$ group B patients experienced at least 1 day of at least mild hypokalaemia after day 1 . Excluding day 1 , there were fewer (although not significantly so) total episodes of hypokalaemia in group A than in group B during the study: 57/143 daily blood samples (maximum of 1 sample per patient per day) (39\%) compared with 80/137 samples $(58 \%)(P=0.21)$. However, there was no difference in the total distribution of CTC toxicity gradings: in group A the proportion of episodes in each grade was $63 \%$ CTC1, $29 \%$ 
(a)
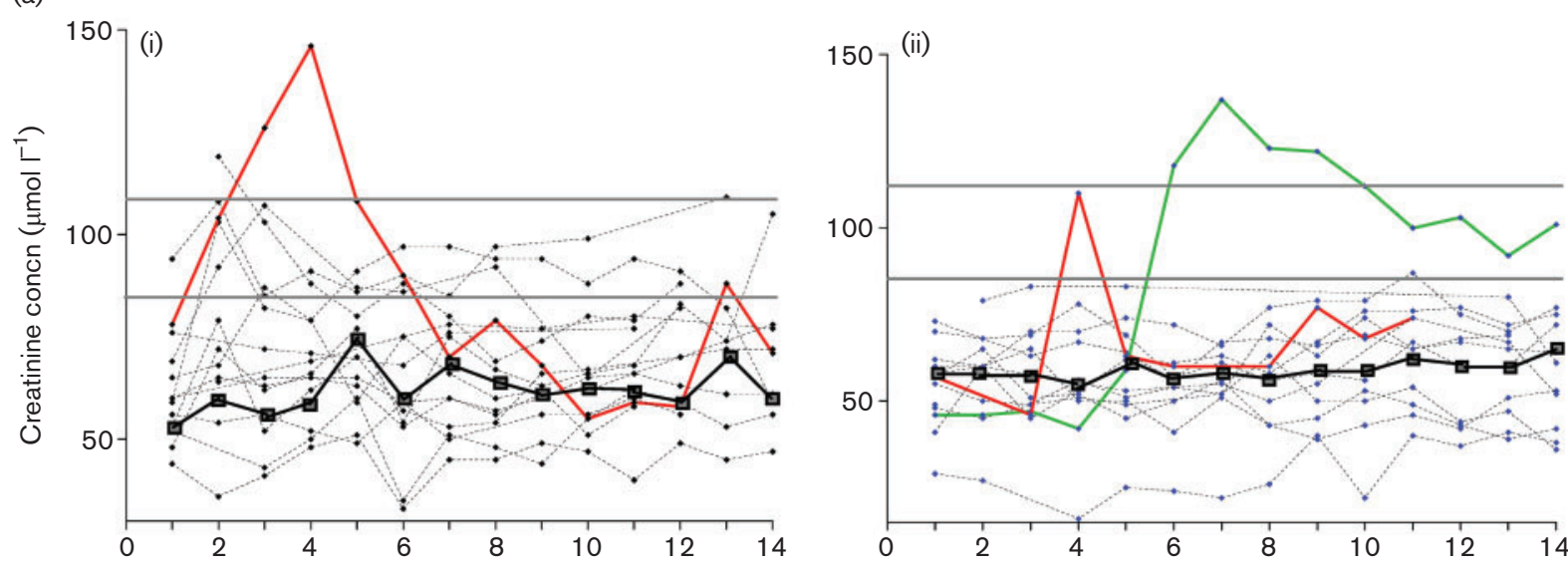

(b)

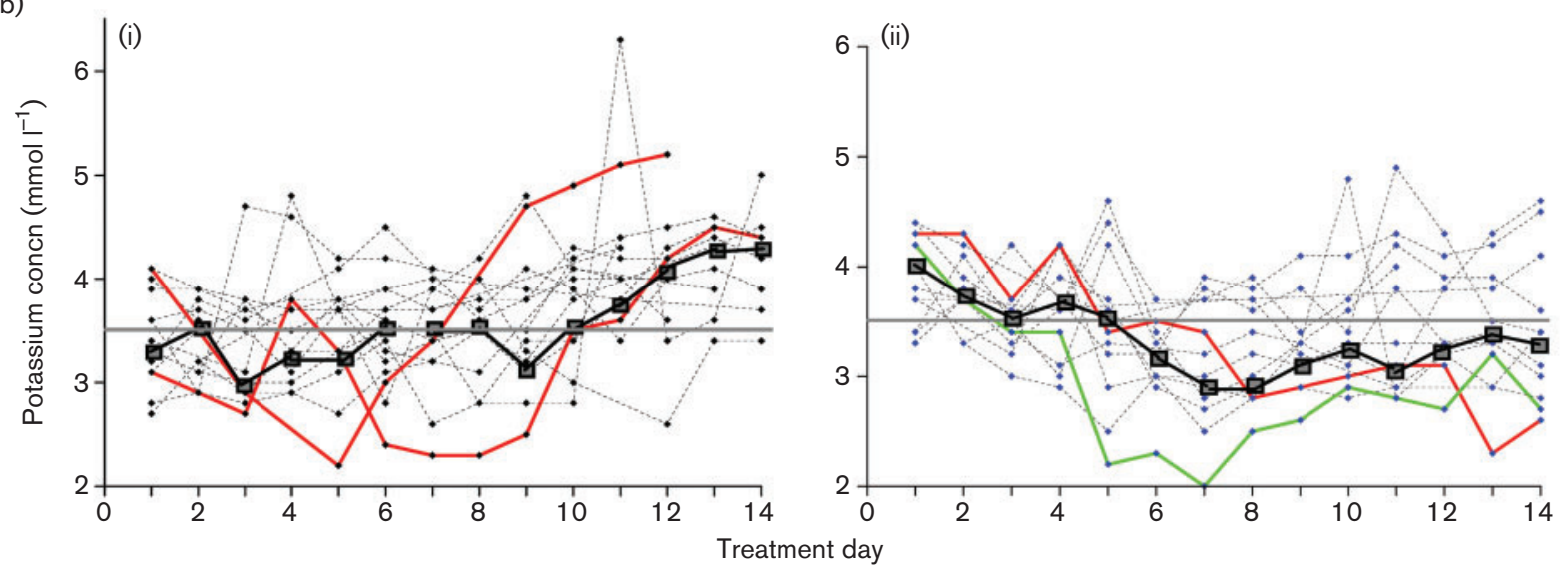

Fig. 1. Individual and median daily concentrations of serum creatinine (a) and potassium (b) for groups A (i) and B (ii). Dashed lines indicate individual subjects. Grey horizontal lines indicate the ULN for males (upper grey line) and females (lower grey line) (a) or the low normal level (b). Coloured lines show the results for individual subjects with persistent raised creatinine (a) or persistent lowered potassium (b) levels. Daily median values are indicated by a solid black line.

CTC3 and $7 \%$ CTC4 compared with group B values of $64 \%$ CTC1, $31 \%$ CTC3 and $5 \%$ CTC4. The lowest value recorded was $2.2 \mathrm{mmol} \mathrm{l}^{-1}$ in one patient in each group.

The serial concentrations of individual patients are depicted in Fig. 1(b). For patients in group A, there was a fall from the baseline low level of $3.4 \mathrm{mmol}^{-1}$ (range 2.7-4.1) over the subsequent 4 days. After day 9 , there was a progressive rise to a normal level of $4.4 \mathrm{mmol} \mathrm{l}^{-1}$ (range 3.4-5.0) at day 14. In contrast, group B patients showed a progressive fall from the baseline and normal value of $3.9 \mathrm{mmol} \mathrm{l}^{-1}$ (range $3.3-4.4$ ) to $2.90 \mathrm{mmol} \mathrm{l}^{-1}$ (range 2.0-3.9) at day 7 and then increased to $3.25 \mathrm{mmol} \mathrm{l}^{-1}$ (range 2.6-4.6) at day 14. The AUC ${ }_{\text {POTASSIUM }}$ for both groups for the first 7 days (excluding baseline) was similar (Supplementary Table S4 available with the online journal). The AUC ${ }_{\text {POTASsIUM }}$ for days $2-14$ was slightly higher in group A (AUC POTASSIUM A:B ratio of 1.06 , $P=0.12$ ).
Fig. 2 shows the proportion of patients with hypokalaemia (CTC1-4) for each day. The proportion of patients with hypokalaemia in group A did not change substantially over the first 7 days, and thereafter fell progressively up to day 14. In contrast, there was a progressive increase in the proportion of patients developing hypokalaemia in group B. By days $7-8,67-84 \%$ of patients had hypokalaemia compared with $31-50 \%$ in group A. Thereafter, this high proportion fell to $46 \%$ at day 13, compared with $12 \%$ in group A. At days 6/7, this was a mean value of $47 \%$ for group A versus $61 \%$ for group B $(P=0.68)$ and for day $12 /$ 13 was $10 \%$ for group A versus $67 \%$ for group B $(P=0.01)$.

Potassium supplements were received by $7 / 15$ patients in group $A$ and $6 / 15$ patients in group $B$, of whom $5 / 15$ versus $1 / 15(P=0.16)$, respectively, received supplements initiated only during the study period. The number of potassiumsupplemented patient days was $64 / 210$ versus $68 / 210$ 


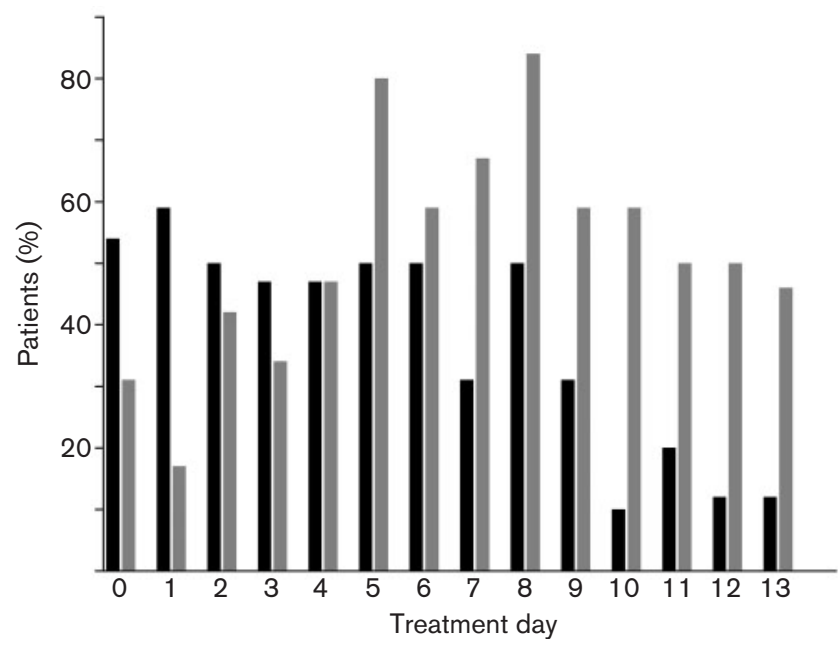

Fig. 2. Proportion of patients with hypokalaemia at CTC1-4 for groups $\mathrm{A}\left(<3.5 \mathrm{mmols}^{-1}\right)$ (black bars) and B (grey bars).

overall, and for those patients commencing supplements during the study period was $41 / 210$ versus $12 / 210(P<0.1)$, respectively, for groups $\mathrm{A}$ and $\mathrm{B}$.

Liver toxicity. The number of patients with persistent rises in at least one liver enzyme or in serum bilirubin was $7 / 15$ (46\%) for group A and 6/15 (40\%) for group B (Supplementary Table S5 available with the online journal). Two patients had other hepatotoxic conditions: one in group A (taking rifampicin + isoniazid for treatment of previous tuberculosis) and one in group B (chronic hepatitis B viral infection being treated with lamivudine). Neither patient had abnormal liver function at baseline.
Bilirubin. Baseline levels were similar in each group (Fig. 3). Median bilirubin levels rose above the ULN during the first 6 days in group A and then returned to normal during the last 7 days. In contrast, the median levels of patients in group B remained close to the ULN throughout. Four patients in group A and two patients in group B had baseline elevated bilirubin levels (CTC1), which fell to within normal range while receiving LAB.

Twelve patients in group A had normal bilirubin levels on most days throughout the study, but four showed transient (1 day) mild (CTC1) elevations. The three other patients in group A (Fig. 3a, coloured lines) had either more persistent or progressive elevations of bilirubin: number 18 with severe erosive herpetic stomatitis (CTC2), number 2 with baseline IPA and chronic hepatitis C virus (HCV) liver disease (CTC3), number 21 with tibial osteomyelitis (CTC1).

In group B, there were also 12 patients with levels close to normal, and transient mild elevations were seen in five of these (Fig. 3b). One further patient (number 11) with raised day 1 bilirubin level sustained a further rise to six times the ULN (CTC3) (this patient had probable IPA and the bilirubin level subsequently fell while continuing LAB). Another four patients sustained rises to twice the ULN (CTC1): number 30 with possible IPA, number 7 with gallstone disease, number 13 with cholecystitis and number 22 with antibiotic drug reaction. The $\mathrm{AUC}_{\text {BILIRUBIN }}$ was not different between groups A and B (Supplementary Table S4 available with the online journal).

Alkaline phosphatase (ALP). Baseline median ALP levels were similar in each group (Supplementary Fig. S2a available with the online journal). Median levels in group A were consistently higher than group B, as reflected by the (a)

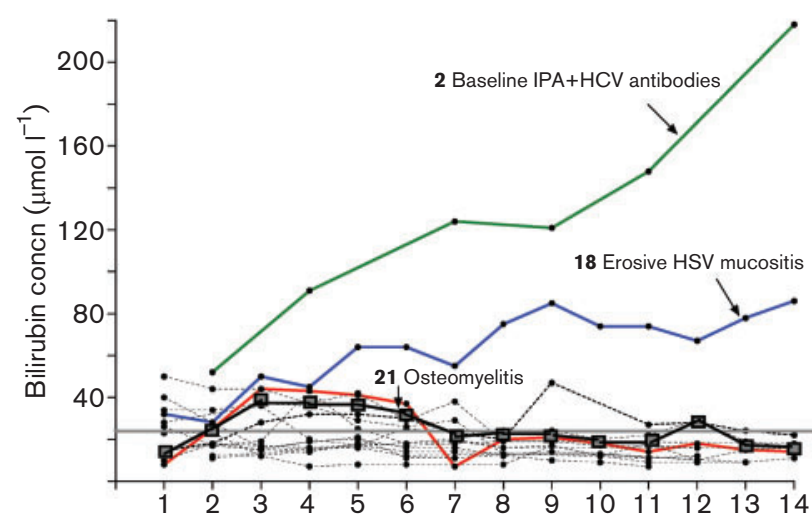

(b)

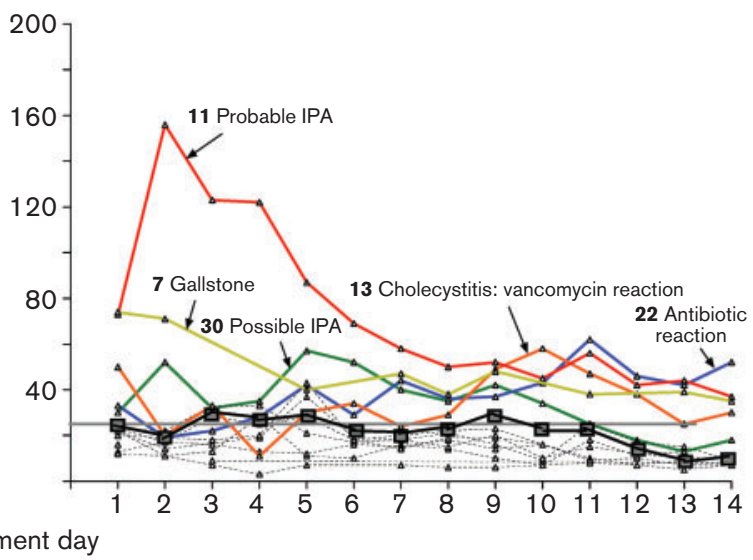

Fig. 3. Individual and median daily serum bilirubin concentrations for groups A (a) and B (b). Dashed lines indicate individual subjects. Grey horizontal lines indicate the ULN. Coloured lines show the results for individual subjects (with patient number indicated) with persistent raised bilirubin. Daily median values are indicated by a solid black line. Identified diagnoses are indicated by arrows. 
higher $\mathrm{AUC}_{\mathrm{ALP}}\left(\mathrm{AUC}_{\mathrm{ALP}} \mathrm{A}: \mathrm{B}\right.$ ratio of $\left.1.19, P=0.12\right)$ for the total 14 day study period (Supplementary Table S4 available with the online journal).

In group $\mathrm{A}$, five patients experienced a progressive rise in ALP to a maximum CTC1; one of these patients had normal baseline levels and four had elevated baseline levels. One further patient had elevated baseline levels that did not improve during the study period. Of the total six patients with high ALP levels, there was no associated co-morbidity in three (numbers 6, 14 and 26), one (number 21) had chronic tibial osteomyelitis, one (number 2) had baseline IPA and chronic HCV infection and one (number 16) had unremittent leukaemia.

In contrast, only one patient (number 30, possible IPA) in group B had a progressive rise in levels, reaching a maximum CTC2. There were two patients with elevated baseline levels in whom the values fell progressively during the study while continuing to receive $\mathrm{LAB}$, one of whom (number 11) had probable IPA and one (number 7) with gallstone disease.

Alanine aminotransferase (ALT). Median ALT levels and $\mathrm{AUC}_{\mathrm{ALT}}$ were similar throughout the study, and within normal levels, in both treatment groups (Supplementary Fig. S2b and Supplementary Table S4 available with the online journal). However, one patient (number 26 with no co-morbidity) in group $\mathrm{A}$ and two patients (number 22 with antibiotic rash; number 30 with possible IPA) in group $\mathrm{B}$ with initially normal levels had increasing elevations to CTC1. Two further patients in each group (group A: number 2 with baseline IPA and HCV; number 4 with no co-morbidity; group B: number 7 with hepatosplenomegaly; number 11 with probable IPA) with initially elevated levels showed normalization during LAB administration.

Aspartate aminotransferase (AST). Median levels were similar in both groups (Supplementary Fig. S2c available with the online journal). In group $\mathrm{A}$, three patients developed mild (CTC1), temporary (1-3 days) elevations. Severe sepsis was identified as a possible factor in one (number 14) of these patients; another (number 18) had severe herpes simplex virus (HSV) disease, whilst one (number 26) had no co-morbidity. One patient (number 2) with baseline IPA and chronic HCV had a high baseline value (CTC2), which fell to CTC1 towards the end of the study period.

A similar profile was documented in group B with three patients sustaining mild and temporary elevations. One patient (number 22) with more sustained high levels (CTC1) had an antibiotic drug reaction. Two patients (number 11 with probable IPA; number 5 with idiopathic liver disease) with high baseline levels (CTC1) (one patient developed probable IPA) fell to within the normal range while receiving $\mathrm{LAB}$. The $\mathrm{AUC}_{\mathrm{AST}}$ for group $\mathrm{A}$ was higher than group $B$ for the first 7 days $\left(\mathrm{AUC}_{\mathrm{AST}} \mathrm{A}: \mathrm{B}\right.$ ratio of 1.36, $P=0.06)$ and for the whole 14 days $\left(\mathrm{AUC}_{\mathrm{AST}} \mathrm{A}: \mathrm{B}\right.$ ratio of $1.37, P=0.03$ ) (Supplementary Table S4 available with the online journal). If the extraordinary patient with very high levels was excluded, the higher ratio was still maintained (first 7 days, $\mathrm{AUC}_{\mathrm{AST}} \mathrm{A}: \mathrm{B}$ ratio of 1.37 , $P=0.06$ ).

Cardiovascular toxicity. There were no significant differences in MAP or maximum and minimum pulse rates throughout the treatment period between the two treatment groups. No patient developed LAB-related hypotension.

\section{Efficacy}

Composite score. Overall success was determined as $11 / 15$ patients $(73 \%)$ in both groups. The individual components of the composite score are shown in Table 2. Breakthrough IFI due to IPA occurred in three patients (two probable, one possible) in group B, diagnosed at days 4, 6 and 14. No patient in group A developed IFI, but one had IPA present at baseline and diagnosed only at day 3 . Three and two patients in groups A and B, respectively, failed to defervesce during neutropenia, although one patient from each group defervesced soon after neutropenia resolution.

There was one death among the 30 patients, within 7 days of completion of LAB treatment in group A. This patient died 4 days after LAB treatment was completed, from progressive leukaemia in the absence of IFI.

Two patients in group A had a change in antifungal treatment for extensive baseline IPA or suspected, subsequently disproven, sinus zygomycosis. One patient in group B had discontinuation of the study drug for failure to respond.

Table 2. Composite success

\begin{tabular}{|lcc|}
\hline & Group A & Group B \\
\hline No. of patients & 15 & 15 \\
Composite success (no. of patients) & 11 & 11 \\
Breakthrough IFI & 0 & $3^{*}$ \\
Resolution of fever in neutropenia & $12 \dagger$ & $13 \dagger$ \\
Survival $>7$ days & $14 \ddagger$ & 15 \\
Successful treatment of baseline IFI & $0 / 1$ & - \\
No discontinuation of treatment & $13 \$$ & $14 \|$ \\
\hline
\end{tabular}

${ }^{\star}$ IPA: two probable, one possible.

$\dagger$ One additional patient in each group defervesced after neutropenia resolution.

$\ddagger$ One death from leukaemia.

§One patient with extensive baseline IPA received $10 \mathrm{mg}$ LAB $\mathrm{kg}^{-1}$ per day; one patient with suspected but disproven sinus zygomycosis received $10 \mathrm{mg} \mathrm{LAB} \mathrm{kg}^{-1}$ per day.

IIOne patient was changed to $5 \mathrm{mg} \mathrm{LAB} \mathrm{kg}^{-1}$ per day for possible IPA. 
BDG concentrations. Excluding patients with either a baseline IFI or who developed an IFI during the study (one in group A and three in group B), the proportion of patients with a positive BDG test in the absence of an IFI at the end of the study were 5/14 (36\%) in group A and 4/12 $(33 \%)$ in group $\mathrm{B}(P=1.00)$. For these 26 patients, the mean $( \pm \mathrm{SD})$ serum BDG concentrations at day 1 were $47.7 \pm 54.1$ and $57.0 \pm 81.4 \mathrm{pg} \mathrm{ml}^{-1}$ for groups $\mathrm{A}$ and $\mathrm{B}$, respectively (Fig. 4). Mean serum BDG levels were somewhat higher at day 9 in both study groups compared with baseline levels, but these increases were not significantly different from the baseline values, or from each other. The $\mathrm{AUC}_{\mathrm{BDG}}$ calculated for all patients apart from the four with either a baseline IFI (one patient) or who developed an IFI (three patients) was 362 and 683 for groups $\mathrm{A}$ and $\mathrm{B}$, respectively $(P=0.36)$.

Defervescence. The rate of fall of fever over the study period did not show a significant difference between the two study groups when tested by a linear mixed model with random intercept and slope $(P=0.75$ for the interaction term).

\section{DISCUSSION}

In this study, LAB given as three separate high doses intermittently over 6 days appeared generally to be as well tolerated as a standard lower dosing regimen. However, any conclusions drawn must be tempered by the small number of subjects recruited, contingent on this being a pilot exploratory study.

The overall proportion of at least one IRADE (40-46\%) is similar to previous published figures of 32-59\% (Leenders et al., 1998; Walsh et al., 2004), as was the $23 \%$ incidence of chills/rigors documented. Furthermore, the overall proportion of administered infusions associated with any IRADE of $9 \%$, or chills/rigors, which accounted for $4.7 \%$,

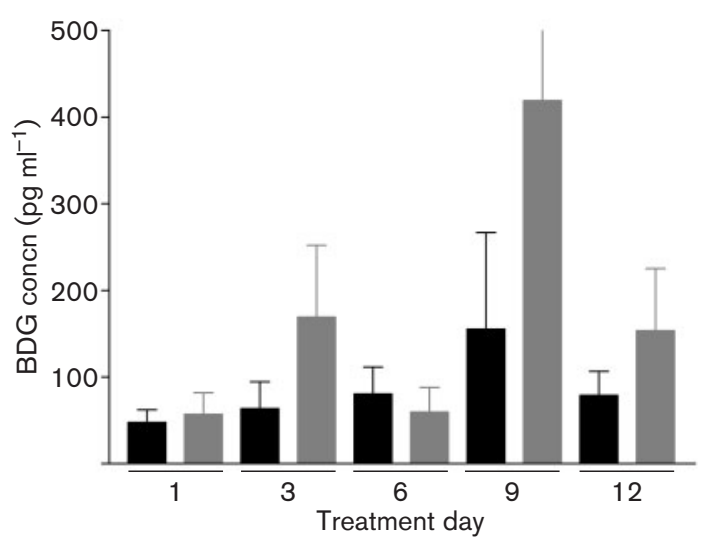

Fig. 4. $B D G$ concentrations in patients without an IFI. Results are shown as means \pm SD for group A (black bars) and group B (grey bars). was also similar to that previously reported (Clark et al., 1998; Mills et al., 1994; Walsh et al., 1999).

However, when analysed as IRADEs associated per infusion, these were significantly higher in patients receiving high-dose LAB compared with the lower, standard dose. Therefore, one in four of the high-dose infusions caused an ADE, half of which were chills/rigors, compared with only one in 17 of the low-dose infusions. This difference does not reflect the disparity in the total number of doses of LAB administered in the two groups, as the large majority of IRADEs occurred during the first three infusions in each group of patients. Our observation of IRADEs, especially chills/rigors, with the higher dose of LAB in group A contrasts with that reported from a multidosing maximum-tolerated-dose study in which day 1 IRADEs were found in $36 \%$ of patients and $14 \%$ of infusions overall (Walsh et al., 2001). Surprisingly, in that study, IRADEs were more common $(62 \%)$ in the $7.5 \mathrm{mg}$ $\mathrm{kg}^{-1}$ group than the $10 \mathrm{mg}(20 \%), 12.5 \mathrm{mg}(29 \%)$ or $15 \mathrm{mg}(37 \%) \mathrm{kg}^{-1}$ groups. Similarly, a study comparing doses of 3 and $10 \mathrm{mg} \mathrm{kg}^{-1}$ per day in IPA did not demonstrate a difference in all ADEs (Cornely et al., 2007). Wingard et al. (2000) did not find a difference in IRADEs between 3 and $5 \mathrm{mg} \mathrm{LAB} \mathrm{kg}{ }^{-1}$ used to treat febrile neutropenic patients. However, in these other studies, the subjects were pre-medicated against chills/rigors in contrast to our study where such medications were given only as required.

One explanation for the excessive frequency of chills/rigors in group A may have been the relatively high rate of infusion for the high concentration of LAB. Previous work has demonstrated that a slower rate of infusion for conventional amphotericin B induces significantly fewer IRADEs (Ellis et al., 1992; Eriksson et al., 2001). The significantly longer infusion rate and increased proportion of patients receiving infusions beyond $150 \mathrm{~min}$ reflected the increased systemic toxicity among group A patients.

Despite the higher IRADE rate per infusion in group A patients, this did not translate into a clinically meaningful disadvantage in terms of treatment discontinuation. As the mean infusion extension time was only $19 \mathrm{~min}$ in group A, no loss in therapeutic efficacy might be expected, in contrast to those situations where infusion times are extended over many hours, where decay of amphotericin B might be anticipated during the infusion or subtherapeutic levels may be maintained throughout (Eriksson et al., 2001).

We assessed both glomerular and tubular nephrotoxicity, with serum creatinine and potassium concentrations, respectively. As published definitions of nephrotoxicity based on creatinine elevations are variable (Saliba \& Dupont, 2008), we also calculated the AUC CREATININE $_{\text {for }}$ the entire study period in addition to the first 7 days, as the latter assessed more closely in time the influence of LAB administration on renal function in group A. 
Using the most common nephrotoxic definition, namely a creatinine rise to $\geqslant 1.5$-fold above the gender-related ULN (NCI, 1998; Saliba \& Dupont, 2008), we found that no patient developed such nephrotoxicity. Also, the AUC $_{\text {CREATININE }}$ was remarkably similar for both groups, for both the 7 and 14 day cumulative periods. Of the three patients with rises to $50 \%$ above the ULN value, explanations other than $\mathrm{LAB}$ were deemed more likely (such as septic shock, antibiotic allergy and pulmonary embolism). This low incidence of LAB-induced hypercreatinaemia in our study contrasts starkly with the 10-50\% incidence of nephrotoxicity reported elsewhere with LAB (Saliba \& Dupont, 2008). Possible explanations may be the low number of concomitant nephrotoxic agents our patients received (a mean of 1.0-1.3 per patient) compared with other studies (Prentice et al., 1997; Walsh et al., 1999) differences in nephrotoxicity definitions, the inclusion of less severely ill patients, the low incidence of important comorbidities likely to influence renal function or the lower number of patients with documented IFIs (Bates et al., 2001; Gubbins et al., 2002).

Nephrotoxicity has been shown to be dose dependent (Walsh et al., 2002, 2004). Cornely et al. (2007) clearly demonstrated an incidence of $27 \%$ when $10 \mathrm{mg}$ LAB $\mathrm{kg}^{-1}$ per day compared with $3 \mathrm{mg} \mathrm{LAB} \mathrm{kg}^{-1}$ per day $(P=0.002)$ was used to treat IPA (Cornely et al., 2007). It is therefore notable that the increased nephrotoxicity expected in group A was not documented, particularly as we did not utilize saline loading, low-dose dopamine or very prolonged infusion times or other debatable 'reno-protective' therapeutic manoeuvres (Camp et al., 1998; Ellis, 2002; Eriksson et al., 2001). However, dose-dependent hypercreatinaemia has not been observed consistently (Walsh et al., 2001). The 24 and $48 \mathrm{~h}$ of infusion-free intervals during administration of the three doses of $\geqslant 5 \mathrm{mg} \mathrm{kg}^{-1}$ was possibly the critical factor preserving patients' renal function in group $A$. The $54 \%$ less cumulative LAB administration in group A may also have obviated renal damage, as higher cumulative doses of amphotericin B are known to be nephrotoxic (Bates et al., 2001).

The overall incidence of hypokalaemia on any day (serum potassium $<3.5 \mathrm{mmol}^{-1}$ ) in this study was high at over $80 \%$. It is not possible to compare nephrotoxicity as defined by this value with other studies because usually only the more severe grades of hypokalaemia have been reported. However, our overall incidence of CTC3 hypokalaemia of $29-31 \%$ is similar to that reported (16$39 \%$ ) in other studies (Saliba \& Dupont, 2008; Walsh et al., 1999, 2001, 2004; Wingard et al., 2000). Despite the higher proportion of patients in group A with hypokalaemia at baseline, tubular toxicity tended to occur less frequently in patients assigned to the high intermittent $\mathrm{LAB}$ dosing, as indicated by the higher $\mathrm{AUC}_{\mathrm{POTASSIUM}}$ in group $\mathrm{A}$ and the higher proportion of patients with hypokalaemia in the standard lower dosing group B. Previous studies, however, have indicated that tubular dysfunction is more often caused by higher LAB doses (Cornely et al., 2007;
Walsh et al., 2001). In the multiple dosing study of Walsh et al. (2001), for example, the incidence of hypokalaemia of $<3.0 \mathrm{mmol} \mathrm{l}^{-1}$ was $37 \%$ at $15 \mathrm{mg} \mathrm{kg}^{-1}$ and $0 \%$ at $7.5 \mathrm{mg}$ $\mathrm{kg}^{-1}$. Our observation of a lower incidence of hypokalaemia associated with a higher LAB dose, however, cannot be unequivocally interpreted as a direct consequence of the limited and intermittent dosing regimen of group $\mathrm{A}$, as this group also received more oral potassium supplementation that was initiated during the study period. This was probably given because of the larger proportion of patients in group A with hypokalaemia at or before day 1. The findings nevertheless suggest that, provided adequate potassium supplementation is given, hypokalaemia associated with short-course, intermittent, high-dose LAB treatment occurs less frequently compared with standard daily dosing.

Biochemical liver damage was similar in the two study groups. Patients with leukaemia often have multiple comorbid conditions that might cause liver damage; indeed, we were able to document at least one such factor in 10 of the 13 patients with liver enzyme abnormalities. This confounds ascribing LAB as a direct cause of the abnormal liver enzymes, with the possible exception of two patients (numbers 26 and 21) in group A who appeared to have a close temporal relationship with the high-dose scheduling (Supplementary Fig. S2a available with the online journal). Despite continuation of LAB, the majority of such patients had resolution of the raised liver enzymes by the end of the study. Even patients with baseline elevations of liver enzymes were noted to improve on $\mathrm{LAB}$ treatment, apart from one case (number 2 in group A). Although some transient elevation of aminotransferases occurred in approximately one-third of patients, our findings were consistent with a very low risk for severe and progressive hepatotoxicity from LAB with either a 3 or $10 \mathrm{mg} \mathrm{kg}^{-1}$ dose (Coukell \& Brogden, 1998; Ellis et al., 2001; Meunier et al., 1991). Our observations also suggest that high-dose LAB given intermittently is as safe as the daily lower dose regimen. Severe LAB-associated hepatoxicity is indeed rare, but may be linked to higher doses (Ellis et al., 2001). The higher AUC $\mathrm{AST}_{\mathrm{AST}}$ observed in group A is consistent with the possibility that patients receiving a higher dose of $\mathrm{LAB}$ might be more at risk from hepatotoxicity. However, in a multiple dosing study, this was not documented, even with doses of up to $15 \mathrm{mg}$ $\mathrm{kg}^{-1}$ (Walsh et al., 2001), and there was no difference in liver enzyme abnormalities in patients receiving 3 or $10 \mathrm{mg} \mathrm{LAB}$ $\mathrm{kg}^{-1}$ per day (Cornely et al., 2007).

Antifungal efficacy related to tissue accumulation and retention of amphotericin $\mathrm{B}$ following intermittent $\mathrm{LAB}$ dosing has been demonstrated in several animal models (Adler-Moore et al., 2004; Garcia et al., 2000; Smith et al., 2007). We have also demonstrated previously that antifungal activity persists in both the serum and tissues for at least several days following intermittent high doses of LAB (Ellis et al., 2007). Furthermore, single or intermittent dosing of LAB has been shown to be feasible (Cordonnier et al., 2005; El-Cheikh et al., 2007), and clinically effective for established 
IFIs (Garbino \& Adam, 2006) and prophylaxis (Kelsey et al., 1999; Mehta et al., 2006; Penack et al., 2006).

The present study also suggests that intermittent high dosing of LAB may be an effective empirical therapy for patients with PFN. However, the study findings, whilst appearing to demonstrate a similar clinical outcome in both groups, should be treated with extreme caution, as our sample size was small, which limits the precise determination of meaningful differences in outcome.

Given this constraint, efficacy of the $10 / 5 / 5 \mathrm{mg} \mathrm{kg}^{-1}$ threedose regimen appears equivalent to the standard $3 \mathrm{mg}$ $\mathrm{kg}^{-1}$ per day dosage, in terms of the five-point composite score, time to defervescence and BDG profiles. Furthermore, although BDG estimates may predict IFIs (Ellis et al., 2008), the use of this assay as a reliable tool for assessing efficacy in PFN patients has not been validated. The high rate of positive BDG tests in each group may reflect early subclinical IFIs that are successfully eradicated by LAB at either dose. Alternatively, it may reflect the low specificity of the test or perhaps mucositis-related fungal antigen leakage (Ellis, 2004, 2008). In addition, there are conflicting opinions as to what should constitute a 'response' to antifungal therapy in a population of PFN patients. Fever resolution, survival and toxicity may be unrelated to response, and breakthrough IFI may be more relevant (Klastersky, 2004; Sobel, 2006).

Although we observed a similar rate of fever resolution in each treatment group, causes of fever other than early IFI, such as bacterial infection, may have been present, hence limiting our ability to ascribe defervescence entirely to antifungal treatment. It is notable in this regard that additional antimicrobials were substituted/added equally to the piperacillin-tazobactam regimen (despite negative blood cultures). Fever resolution may also have depended partially on white cell recovery, although this was similar for each treatment group.

In conclusion, this pilot exploratory study suggests that high-dose, short-course, intermittent dosing with LAB in patients with PFN is as safe and effective as a standard lower dose over a longer period, but with the advantages of reduced drug exposure, hence limiting costs and possibly toxicities. A larger study is therefore indicated.

\section{ACKNOWLEDGEMENTS}

We are grateful for Gilead Sciences for an unrestricted scientific grant. We thank Mrs Mary Jacobs, the Research Nurse involved in this study. We thank the Nursing Team at Tawam-Johns Hopkins Hospital for their support.

\section{REFERENCES}

Adler-Moore, J. \& Proffitt, R. T. (2003). Effect of tissue penetration on AmBisome efficacy. Curr Opin Investig Drugs 4, 179-185.

Adler-Moore, J. P. \& Proffitt, R. T. (2008). Amphotericin B lipid preparations: what are the differences? Clin Microbiol Infect 14 (Suppl. 4), 25-36.
Adler-Moore, J. P., Olson, J. A. \& Proffitt, R. T. (2004). Alternative dosing regimens of liposomal amphotericin B (AmBisome) effective in treating murine systemic candidiasis. J Antimicrob Chemother 54, 1096-1102.

Bates, D. W., Su, L., Yu, D. T., Chertow, G. M., Seger, D. L., Gomes, D. R., Dasbach, E. J. \& Platt, R. (2001). Mortality and costs associated with amphotericin B therapy. Clin Infect Dis 32, 686-693.

Bekersky, I., Fielding, R. M., Dressler, D. E., Lee, J. W., Buell, D. N. \& Walsh, T. J. (2002). Pharmacokinetics, excretion, and mass balance of liposomal amphotericin B (AmBisome) and amphotericin B deoxycholate in humans. Antimicrob Agents Chemother 46, 828-833.

Camp, M. J., Wingard, J. R., Gilmore, C. E., Lin, L. S., Dix, S. P., Davidson, T. G. \& Geller, R. B. (1998). Efficacy of low dose dopamine in preventing amphotericin B nephrotoxicity in bone marrow transplant patients and leukemia patients. Antimicrob Agents Chemother 42, 3103-3106.

Clark, A. D., McKendrick, S., Tansey, P. J., Franklin, I. M. \& Chopra, R. (1998). A comparative analysis of lipid-complexed and liposomal amphotericin B preparations in haematological oncology. $\mathrm{Br} J$ Haematol 103, 198-204.

Coiffier, B., Lepage, E., Briere, J., Herbrecht, R., Tilly, H., Bouabdallah, R., Morel, P., Van Den Neste, E., Salles, G. \& other authors (2002). CHOP chemotherapy plus rituximab compared with CHOP alone in elderly patients with diffuse large-B-cell lymphoma. $N$ Engl J Med 346, 235-242.

Collette, N., Van der Auwera, P., Meunier, F., Lambert, C., Sculier, J. P. \& Coune, A. (1991). Tissue distribution and bioactivity of amphotericin B administered in liposomes to cancer patients. J Antimicrob Chemother 27, 535-548.

Cordonnier, C., Mohty, M., Faucher, C., Vey, N., Pautas, C., Robin, M., Mahi, L. \& Ribaud, P. (2005). Safety of a weekly administration of $10 \mathrm{mg} / \mathrm{kg}$ of liposomal amphotericin B for antifungal prophylaxis in allogeneic stem-cell transplant and acute leukemia patients. In American Society of Hematology 47th Annual Meeting, 10-13 December 2005, Atlanta, GA, USA, abstract 5346.

Cornely, O. A., Maertens, J., Bresnik, M., Ebrahimi, R., Ullmann, A. J., Bouza, E., Heussel, C. P., Lortholary, O., Rieger, C. \& other authors (2007). Liposomal amphotericin B as initial therapy for invasive mold infection: a randomized trial comparing a high-loading dose regimen with standard dosing (AmBiload Trial). Clin Infect Dis 44, 1289-1297.

Coukell, A. J. \& Brogden, R. N. (1998). Liposomal amphotericin B: therapeutic use in the management of fungal infections and visceral leishmaniasis. Drugs 55, 585-612.

Demartini, G., Lequaglie, C., Brega Messone, P. P., Scaglione, F. \& Fraschini, F. (2005). Penetration of amphotericin B in human lung tissue after single liposomal amphotericin B (AmBisome) infusion. J Chemother 17, 82-85.

De Pauw, B., Walsh, T. J., Donnelly, J. P., Stevens, D. A., Edwards, J. E., Calandra, T., Pappas, P. G., Maertens, J., Lortholary, O. \& other authors (2008). Revised definitions of invasive fungal disease from the European Organisation for Research and Treatment of Cancer/ Invasive Fungal Infections Cooperative Group and the National Institute of Allergy and Infectious Diseases Mycoses Study Group (EORTC/MSG) Consensus Group. Clin Infect Dis 46, 1813-1821.

Deray, G. (2002). Amphotericin B nephrotoxicity. J Antimicrob Chemother 49 (Suppl. 1), 37-41.

El-Cheikh, J., Faucher, C., Furst, S., Duran, S., Berger, P., Vey, N., Stoppa, A. M., Bouabdallah, R. \& other authors (2007). High dose weekly liposomal amphotericin B antifungal prophylaxis following reduced intensity conditioning allogeneic stem cell transplantation. Bone Marrow Transplant 39, 301-306. 
Ellis, M. (2002). Invasive fungal infections: evolving challenges for diagnosis and therapeutics. Mol Immunol 38, 947-957.

Ellis, M. (2004). Preventing microbial translocation in haematological malignancy. Br J Haematol 125, 282-293.

Ellis, M. (2008). New dosing strategies for liposomal amphotericin B in high-risk patients. Clin Microbiol Infect 14 (Suppl. 4), 55-64.

Ellis, M. E., al-Hokail, A., Clink, H., Padmos, A., Ernst, P., Spence, D. G., Tharpe, W. N. \& Hillier, V. F. (1992). Double blind randomized study of the effect of infusion rates on toxicity of amphotericin B. Antimicrob Agents Chemother 36, 172-179.

Ellis, M., Shamoon, A., Gorka, W., Zwaan, F. \& al-Ramadi, B. (2001). Severe hepatic injury associated with lipid formulations of amphotericin B. Clin Infect Dis 32, E87-E89.

Ellis, M., Hedstrom, U., al-Ramadi, B., Alizadeh, H., Kristensen, J., Kshirsagar, S., Blaschke, T., Stevens, D., Klingspor, L. \& Poughias, L. (2007). Pharmacokinetics, efficacy of $3 \mathrm{mg} / \mathrm{kg} /$ day vs $10 \mathrm{mg} / \mathrm{kg}$ day 1,5 $\mathrm{mg} / \mathrm{kg}$ days 3 and 6 liposomal amphotericin B (Ambisome) in febrile neutropenia. In 8th Congress of the European Association for Clinical Pharmacology and Therapeutics, 29 August-1 September 2007, Amsterdam, the Netherlands, abstract P257.

Ellis, M., al-Ramadi, B., Finkelman, M., Hedstrom, U., Kristensen, J., Ali-Zadeh, H. \& Klingspor, L. (2008). Assessment of the clinical utility of serial $\beta$-D-glucan concentrations in patients with persistent neutropenic fever. J Med Microbiol 57, 287-295.

EORTC IATCG (1989). Empiric antifungal therapy in febrile granulocytopenic patients. Am J Med 86, 668-672.

Eriksson, U., Seifert, B. \& Scaffner, A. (2001). Comparison of effects of amphotericin B deoxycholate infused over 4 or 24 hours: randomized controlled trial. BMJ 322, 579-582.

Farag, S. S., Ruppert, A. S., Mrozek, K., Mayer, R. J., Stone, R. M., Carroll, A. J., Powell, B. L., Moore, J. O., Pettanati, M. J. \& other authors (2005). Outcome of induction and post-remission therapy in younger adults with acute myeloid leukemia with normal karyotype: a cancer and leukemia group study. J Clin Oncol 23, 482-493.

Fleiss, J., Levin, B. \& Paik, M. (2003). Statistical Methods for Rates and Proportions, 3rd edn. New York: Wiley Interscience.

Francis, P., Lee, J. W., Hoffman, A., Peter, J., Francesconi, A., Bacher, J., Shelhamer, J., Pizzo, P. A. \& Walsh, T. J. (1994). Efficacy of unilamellar liposomal amphotericin B in treatment of pulmonary aspergillosis in persistently granulocytopenic rabbits: the potential role of brochoalveolar D-mannitol and serum galactomannan as markers of infection. J Infect Dis 169, 356-368.

Garbino, J. \& Adam, A. (2006). Use of high-dose liposomal amphotericin B: efficacy and tolerance. Acta Biomed 77 (Suppl. 4), 19-22.

Garcia, A., Adler-Moore, J. \& Proffitt, R. (2000). Single-dose AmBisome (liposomal amphotericin B) as prophylaxis for murine systemic candidiasis and histoplasmosis. Antimicrob Agents Chemother 44, 2327-2332.

Garey, K. W., Rege, M., Pai, M. P., Mingo, D. E., Suda, K. J., Turpin, R. S. \& Bearden, D. T. (2006). Time to initiation of fluconazole therapy impacts mortality in patients with candidemia: a multiinstitutional study. Clin Infect Dis 43, 25-31.

Goldberg, E., Gafter-Gvili, A., Robenshtok, E., Leibovici, L. \& Paul, M. (2008). Empirical antifungal therapy for patients with neutropenia and persistent fever: systematic review and meta-analysis. Eur J Cancer 44, 2192-2203.

Greene, R. E., Schlamm, H. T., Oestmann, J. W., Stark, P., Durand, C., Lortholary, O., Wingard, J. R., Herbrecht, R., Ribaud, P. \& other authors (2007). Imaging findings in acute invasive pulmonary aspergillosis: clinical significance of the halo sign. Clin Infect Dis 44, 373-379.

Gubbins, P. O., Penzak, S. R., Ploston, S., McConnell, S. A. \& Anaissie, A. (2002). Characterizing and predicting amphotericin
B-associated nephrotoxicity in bone marrow or peripheral blood stem cell transplant recipients. Pharmacotherapy 22, 961-971.

Hughes, W. T., Armstrong, D., Bodey, G. P., Bow, E. J., Brown, A. E., Calandra, T., Feld, R., Pizzo, P. A., Rolston, K. V. I. \& other authors (2002). 2002 guidelines for the use of antimicrobial agents in neutropenic patients with cancer. Clin Infect Dis 34, 730-751.

Kelsey, S. M., Goldman, J. M., McCann, S., Newland, A. C., Scarffe, J. H., Oppenheim, B. A. \& Mufti, G. J. (1999). Liposomal amphotericin $B$ (AmBisome) in the prophylaxis of fungal infections in neutropenic patients: a randomized, double-blind placebo-controlled study. Bone Marrow Transplant 23, 163-168.

Klastersky, J. (2004). Antifungal therapy in patients with fever and neutropenia - more rational and less empirical? N Engl J Med 351, 1445-1447.

Lanternier, F. \& Lortholary, O. (2008). Liposomal amphotericin B: what is its role in 2008? Clin Microbiol Infect 14 (Suppl. 4), 71-83.

Leenders, A. C., Daenen, S., Jansen, R. L., Hop, W. C., Lowenberg, B., Wijermans, P. W., Cornelissen, J., Herbrecht, R. \& other authors (1998). Liposomal amphotericin B compared with amphotericin B deoxycholate in the treatment of documented and suspected neutropenia-associated invasive fungal infections. $\mathrm{Br} J$ Haematol 103, 205-212.

Marr, K. A. (2008). Primary antifungal prophylaxis in hematopoietic stem cell transplant recipients: clinical implications of recent studies. Curr Opin Infect Dis 21, 409-414.

Masiá Canuto, M. M. \& Gutiérrez Rodero, F. (2002). Antifungal drug resistance to azoles and polyenes. Lancet Infect Dis 2, 550-563.

MRC WPLA (2006). Medical Research Council Acute Lymphoblastic Leukemia Trial XII (UKALL XII), version 4.1, February 2006. Joint Trial with Eastern Cooperative Group (ECOG) - E2993. Protocol for adults with ALL. Modified for intrathecal therapy and Philadelphia Positive ALL, July 2002. Revised May 2005, February 2006. http:// www.ctsu.ox.ac.uk/projects/leuk/ukall2003

Mehta, P., Vinks, A., Filipovitch, A., Vaughan, G., Fearing, D., Sper, C. \& Davies, S. (2006). High dose weekly AmBisome antifungal prophylaxis in pediatric patients undergoing hematopoietic stem cell transplantation: a pharmacokinetic study. Biol Blood Marrow Transplant 12, 235-240.

Meunier, F., Prentice, H. G. \& Ringden, O. (1991). Liposomal amphotericin B (AmBisome): safety data from a phase II/III clinical trial. J Antimicrob Chemother 28 (Suppl. B), 83-91.

Mills, W., Chopra, R., Linch, D. C. \& Goldstone, A. H. (1994). Liposomal amphotericin B in the treatment of fungal infections in neutropenic patients: a single centre experience of 133 episodes in 116 patients. Br J Haematol 86, 754-760.

Moen, M. D., Lyseng-Williamson, K. A. \& Scott, L. J. (2009). Liposomal amphotericin B: a review of its use as empirical therapy in febrile neutropenia and in the treatment of invasive fungal infections. Drugs 69, 361-392.

NCl (1998). Common Toxicity Criteria, version 2.0. Bethesda, MD: National Cancer Institute. http://ctep.cancer.gov/protocolDevelopment/ electronic_applications/docs/ctcv20_4-30-992.pdf.

Olson, J. A., Adler-Moore, J., Schwartz, J., Jensen, G. \& Proffitt, R. (2006). Comparative efficacies, toxicities, and tissue concentrations of amphotericin B lipid formulations in a murine aspergillosis model. Antimicrob Agents Chemother 50, 2122-2131.

Ostrosky-Zeichner, L., Marr, K. A., Rex, J. H. \& Cohen, S. H. (2003). Amphotericin B: time for a new 'gold standard'. Clin Infect Dis 37, 415-425.

Patterson, T. F., Kirkpatrick, W. R., White, M., Hiemenz, J. W., Wingard, J. R., Dupont, B., Rinaldi, M. G., Stevens, D. A. \& Graybill, J. R., for the Aspergillus Study Group (2000). Invasive aspergillosis: 
disease spectrum, treatment practices, and outcomes. Medicine (Baltimore) 79, 250-260.

Penack, O., Schartz, S., Martus, P., Reinwald, M., Schmidt-Hieber, M., Thiel, E. \& Blau, I. W. (2006). Low dose liposomal amphotericin B in the prevention of invasive fungal infections in patients with prolonged neutropenia: results from a randomized single center trial. Ann Oncol 17, 1306-1312.

Prentice, H. G., Hann, I. M., Herbrecht, R., Aoun, M., Kvaloy, S., Catovsky, D., Pinkerton, C. R., Schey, S. A., Jacobs, F. \& other authors (1997). A randomized comparison of liposomal versus conventional amphotericin $\mathrm{B}$ for the treatment of pyrexia of unknown origin in neutropenic patients. Br J Haematol 98, 711-718.

Saliba, F. \& Dupont, B. (2008). Renal impairment and amphotericin B formulations in patients with invasive fungal infections. Med Mycol 46, 97-112.

Smith, P. J., Olson, J. A., Constable, D., Schwartz, J., Proffitt, R. T. \& Adler-Moore, J. (2007). Effects of dosing regimen on accumulation, retention and prophylactic efficacy of liposomal amphotericin B. J Antimicrob Chemother 59, 941-951.

Sobel, J. D. (2006). Design of clinical trials of empiric antifungal therapy in patients with persistent febrile neutropenia: considerations and critiques. Pharmacotherapy 26, 47S-54S.

Takemoto, K., Yamamoto, Y., Ueda, Y., Sumita, Y., Yoshida, K. \& Niki, Y. (2006a). Comparative study on the efficacy of AmBisome and Fungizone in a mouse model of pulmonary aspergillosis. J Antimicrob Chemother 57, 724-731.

Takemoto, K., Yamamoto, Y. \& Ueda, Y. (2006b). Evaluation of antifungal pharmacodynamic characteristics of AmBisome against Candida albicans. Microbiol Immunol 50, 579-586.

Townsend, R. W., Zutshi, A. \& Behersky, I. (2001). Biodistribution of $4-\left[{ }^{14} \mathrm{C}\right]$ cholesterol-AmBisome following a single intravenous administration to rats. Drug Metab Dispos 29, 681-685.
Vogelsinger, H., Weiler, S., Djanani, A., Kountchev, J., BellmannWeiler, R., Wiedermann, C. J. \& Bellmann, R. (2006). Amphotericin B distribution in autopsy material after treatment with liposomal amphotericin B and amphotericin B colloidal dispersion. J Antimicrob Chemother 57, 1153-1160.

Walsh, T. J., Finberg, R. W., Arndt, C., Hiemenz, J., Schwartz, C., Bodensteiner, D., Pappas, P., Seibel, N., Greenberg, R. N. \& other authors (1999). Liposomal amphotericin B for empirical therapy in patients with persisting fever and neutropenia. N Engl J Med 340, 764-771.

Walsh, T. J., Goodman, J. L., Pappas, P., Bekersky, I., Buell, D. N., Roden, M., Barrett, J. \& Anaissie, A. J. (2001). Safety, tolerance and pharmacokinetics of high-dose liposomal amphotericin B (AmBisome) in patients infected with Aspergillus species and other filamentous fungi: maximum tolerated dose study. Antimicrob Agents Chemother 45, 3487-3496.

Walsh, T. J., Pappas, P., Winston, D. J., Lazarus, H. M., Petersen, F., Raffalli, J., Yanovich, S., Stiff, P., Greenberg, R. \& other authors (2002). Voriconazole compared with liposomal amphotericin B for empirical antifungal therapy in patients with neutropenia and persistent fever. $N$ Engl J Med 346, 225-234.

Walsh, T. J., Teppler, H., Donowitz, G. R., Maertens, J. A., Baden, L. R., Dmoszynska, A., Cornely, O. A., Bourque, M. R., Lupinacci, R. J. \& other authors (2004). Caspofungin versus liposomal amphotericin B for empirical antifungal therapy in patients with persistent fever and neutropenia. N Engl J Med 351, 1391-1402.

Wingard, J. R., White, M. H., Anaissie, E., Raffalli, J., Goodman, J., Arrieta, A. L. \& Amph/ABLC Collaborative Study Group (2000). A randomized double-blind comparative trial evaluating the safety of liposomal amphotericin B versus amphotericin B lipid complex in the empirical treatment of febrile neutropenia. Clin Infect Dis 31, 1155-1163. 